\title{
Dentine hypersensitivity: analysis of self-care products $\$$
}

\author{
Cassiano Kuchenbecker Rösing ${ }^{(a)}$ \\ Tiago Fiorini ${ }^{(b)}$ \\ Diego Nique Liberman ${ }^{(b)}$ \\ Juliano Cavagni ${ }^{(b)}$
}

(a) Professor of Periodontology; (b) Graduate Student, Graduate Program in Dentistry - School of Dentistry, Federal University of Rio Grande do Sul, Porto Alegre, RS, Brazil.

\begin{abstract}
Dentine hypersensitivity is a condition that is often present in individuals, leading them to seek dental treatment. It has been described as an acute, provoked pain that is not attributable to other dental problems. Its actual prevalence is unknown, but it is interpreted as very unpleasant by individuals. Several therapeutic alternatives are available to manage dentine hypersensitivity, involving both in-office treatment and home-use products. The aim of this literature review was to evaluate selfcare products for managing dentine hypersensitivity. Among the products available, dentifrices and fluorides are the most studied self-care products, with positive effects. However, a high percentage of individuals is affected by the placebo effect. Among dentifrices, those containing potassium salts seem to be the most promising. Dental professionals need to understand the advantages and limitations of these therapies and use this knowledge in a positive approach that might help in decreasing dentine hypersensitivity among patients.
\end{abstract}

Descriptors: Dentin hypersensitivity; Review; Dental devices, home-care.
§Paper presented at the "Oral Health Self-Care Products: Realities and Myths" international symposium, sponsored by the Brazilian Association for Oral Health Promotion (ABOPREV), September 25-27, 2008, São Paulo, SP, Brazil.

\section{Corresponding author:}

Cassiano Kuchenbecker Rösing

Rua Dr. Valle, 433/801

Porto Alegre - RS - Brazil

CEP: 90560-010

E-mail: ckrosing@hotmail.com 


\section{Introduction}

Dentine hypersensitivity is characterized by an acute, short-duration pain related to exposed dentine in response to typically thermal, tactile, osmotic, chemical or evaporative stimuli that cannot be attributed to any other pathological condition. ${ }^{1}$ It is a very unpleasant condition, limiting the daily habits of patients, such as the possibility of eating/drinking different kinds of foods and drinks. It is frequently one of the main reasons for visiting a dentist.

As any other pain sensation in the body, tooth hypersensitivity is viewed by individuals as an important health problem, sometimes leading to an increased concern about their health status. Despite its acute character, and because it is not spontaneous but rather stimulated, dentine hypersensitivity can be considered a chronic pain condition. With this in mind, dental professionals need to develop strategies for managing dentine hypersensitivity, thus improving the quality of life of individuals.

The reported prevalence of dentine hypersensitivity presents a large variation, depending on the target population, on the methods employed to asses tooth hypersensitivity and on analysis of the data. For example, after a clinical evaluation of more than 3,000 individuals in the United Kingdom, Rees ${ }^{2}$ (2000) reported a prevalence of $4 \%$. On the other hand, studies by Chabanski et al. ${ }^{3}$ (1997) and Orchardson, Collings ${ }^{4}$ (1987), also in the United Kingdom, reported prevalences of $73 \%$ and $74 \%$, respectively. However, these two latter studies comprised less than 100 individuals each.

In Brazil, Fischer et al. ${ }^{5}$ (1992), in a University Clinic, reported a prevalence of $25 \%$ of self-reported tooth hypersensitivity, and $17 \%$ were confirmed after clinical examination. This scenario indicates that whatever the real prevalence might be, dentine hypersensitivity is an important problem that needs management, especially because pain is involved.

Different agents have been studied for the prevention and treatment of tooth hypersensitivity. Home-care and in-office approaches have been proposed. The aim of the present study was to perform a review of the literature concerning the use of home-care products in the management of dentine hypersensitivity.

\section{Mechanisms associated with pain sensation}

Dentin is normally sensitive to external stimuli since it has structural and functional relationships with the dental pulp. ${ }^{6}$ However, tooth hypersensitivity is observed when the dentinal tubules of a vital tooth are exposed and subjected to mechanical, chemical and/or thermal stimuli. ${ }^{1}$ Owing to its thin cementum layer, the cervical area generally has dentinal tubules that are exposed by toothbrushing, dietary erosion ${ }^{7}$ and abrasive dentifrices. ${ }^{8}$ This area is responsible for more than $90 \%$ of hypersensitive surfaces. ${ }^{4}$ Several theories have been proposed to explain the pain mechanisms of this condition: the transduction theory, the modulation theory, the gate control and vibration theory and the currently most accepted hypothesis, the hydrodynamic theory.

The hydrodynamic theory was developed in the 1960's and suggests that a stimulus could remove fluid from the dentinal tubules causing a rapid outward flow and a pressure change across the dentine, thus resulting in the activation of pain fibers, mainly A-delta fibers, on the pulp wall. ${ }^{9}$ Dentin covered with smear layer or cementum can be less sensitive than a freshly exposed dentin surface. Similarly, the number and the width of exposed tubules and the presence of sclerotic dentin can influence the intensity of the pain caused by an exogenous stimulus. ${ }^{10-14}$

\section{Factors that may enhance pain sensation}

Some factors can clearly contribute or even potentiate the development of tooth hypersensitivity. The most common clinical event related to the occurrence of dentine hypersensitivity is gingival recession, when the cervical area becomes apparent and the exposure of dentinal tubules is observed because of a thin cementum layer. A retrospective study with a representative sample of an urban Brazilian population ${ }^{15}$ revealed that more than half $(51.6 \%)$ and $22.0 \%$ of individuals presented gingival recession $\geq 3 \mathrm{~mm}$ and $\geq 5 \mathrm{~mm}$, respectively. This condition was primarily related to destructive periodontal disease and was associated with smoking and the presence of supragingival calculus.

In patients submitted to periodontal treatment, 
tooth hypersensitivity is frequently correlated to the procedures of scaling and root planing, which remove the cementum layer from the root surface. ${ }^{5}$ Chabanski et al. ${ }^{3}$ (1997) described a prevalence of tooth hypersensitivity varying from $72.5 \%$ to $98 \%$ of individuals after periodontal therapy. More recently, a systematic review ${ }^{16}$ revealed that the prevalence of tooth hypersensitivity ranged from $9 \%$ to $23 \%$ before periodontal treatment and from $54 \%$ to $55 \%$ after it.

Another factor that can potentiate this condition is the individual's diet, especially after exposure to acidic drinks or fruits like lemon. When Prati et al. ${ }^{17}$ (2003) evaluated dentin permeability and morphology after erosion in the presence of acidic drinks, they found an increased dentin permeability caused by the dissolution of the smear layer and smear plugs and, consequently, an increased risk for dentin hypersensitivity. Because the exposure of dentinal tubules is involved, studies performed in beagle $\operatorname{dog} \mathrm{s}^{18}$ and in situ ${ }^{19}$ concluded that a good plaque control, performed mechanically or chemically, can reduce the patency of dentinal tubules by occluding them in a natural reparative process of tooth hypersensitivity.

On the other hand, plaque control performed with an abrasive dentifrice can result in increased patency of dentinal tubules. The use of a less-abrasive dentifrices is able to prevent dentin hypersensitivity in cervical regions. ${ }^{20}$ Another current topic is that tooth hypersensitivity has been the most commonly reported adverse effect of vital tooth whitening with peroxide gels, and its complex etiology has been the major obstacle to define prevention strategies. ${ }^{21}$

Other pain mechanisms are possibly involved in the occurrence of tooth hypersensitivity and may be related to nerve activation instead of, or in addition to, the hydrodynamic mechanism. Inflammation may sensitize the nerve endings to an extent that smaller fluid shifts would be sufficient for nerve activation. ${ }^{22}$ Since inflammatory mediators can increase the sensitivity of the responding nerve fibers, leading to a poor plaque control, ${ }^{23}$ the constant inflammatory state would result in the presence of inflammatory mediators that could result in a vicious circle for dentin hypersensitivity.

During patient anamnesis, it is important for the clinician to identify factors that can potentiate tooth hypersensitivity. This information can be important in choosing a correct approach to change habits and in treatment success.

\section{Difficulties and limitations in the study of dentine hypersensitivity}

Pain is a subjective experience and depends on various factors, such as the psychological profile of the individual, his/her previous pain experiences and levels of anxiety. Therefore, great intra- and inter-individual variations are common in pain studies and, consequently, in tooth hypersensitivity studies. Different forms of stimulation are associated with varying results. One of the most common stimuli used in dentine hypersensitivity studies is a blast of air during one second. Even this method, apparently simple and reliable, has been demonstrated to vary quite importantly. The temperature of the air varies according to the external temperature and this variation may or may not exceed individual thresholds, resulting in distinct responses. Moreover, the air pressure, the distance from the source of application and the time of stimulation may vary, thus giving different intensities of the stimulus. ${ }^{24}$

Concerning the quantification of pain, in some studies it is performed with a verbal rating scale (VRS) with 4 degrees of intensity, which may limit the options for the patient to describe the pain and lead to less consistent results in the clinical observation. Other aspects that can make the interpretation of alleviating effects of desensitizing agents difficult are the Hawthorne effect and the placebo effect (which will be discussed further). In many cases, spontaneous healing of the pain is observed, even without any treatment approach. These difficulties and limitations, together with the subjectivity of the pain sensation, sometimes lead to inconclusive and contradictory results. ${ }^{25}$

Studies that address dentine hypersensitivity present a high degree of heterogeneity in terms of methods, especially concerning the selection of volunteers and time of follow-up. These differences, together with the high diversity of studied agents, sometimes make interpretation of the evidence very laborious or even impossible. The number of ran- 
domized controlled clinical trials with adequate numbers of individuals and sufficient follow-up intervals is still low. Taking into consideration the difficulties and limitations in the study of dentine hypersensitivity, the real magnitude of the effects of the substances available to treat it is uncertain.

\section{Fluorides and dentine hypersensitivity}

The use of fluorides for treating dentine hypersensitivity has been widely proposed in the literature. $^{26-28}$ The main objective is to deposit fluoride on the dental surfaces, especially in the dentinal tubules, causing their occlusion in a manner that external stimuli would be prevented from reaching the dental pulp. Fluorides have been utilized under the supervision of dentists, with high concentration products, as well as by the patient, with low fluoride concentration dentifrices and mouthrinses. The use of high dose fluorides must be performed with caution to avoid acute toxicity. Patients should also be acquainted with the risks of intoxication in case of ingestion by children. Acute effects of intoxication include gastrointestinal, neurological, and cardiovascular symptoms, ranging from a little distress to death. Chronic side effects are found in mineralized tissues, leading to dental or skeletal fluorosis, depending on the dose. ${ }^{29,30}$

Clinical trials have been published with the aim of testing which is the best agent for treating tooth hypersensitivity, ${ }^{31-33}$ but most of them did not use a placebo control group. Case series are an interesting form of generating hypotheses, but the evidence from this kind of study is not unequivocal. Comparison is therefore extremely necessary, since the placebo effect has been demonstrated to be an important bias in clinical trials, especially when pain is concerned.

One of the few randomized, double-blind, placebo controlled clinical trials published in the related literature up to the present moment was performed by Yates et al. ${ }^{34}$ (2004). The results showed no significant improvement in dentine hypersensitivity when the test and placebo groups were compared 56 days after treatment. It therefore remains unclear why high prevalence rates of tooth hypersensitivity are found among populations that use fluoride dentifrices constantly and live in fluoridated water regions. ${ }^{25}$

Hence, only low-quality evidence is available showing an additional benefit of the use of fluorides in reducing hypersensitivity when compared to a placebo. This does not mean that the use of fluorides must be abandoned and the use of a placebo recommended. The agent can be used as an adjunct to the treatment, together with a positive approach by the dental professional.

\section{Dentifrices for treating dentine hypersensitivity}

A treatment for dentine hypersensitivity, as well as for other problems, should ideally be performed in a short period of time, and it should be cheap and easy to perform. Thus, the use of dentifrices has been proposed as one of the first alternatives. They are non-invasive, easily available (in drugstores and supermarkets) and have a good cost-benefit relationship, especially if compared to professional appointments. Additionally, toothbrushing is a common habit both in developed and developing countries, and does not involve additional requirements on the part of the patient.

Together with fluoride, that has been previously discussed as an effective agent for treating tooth hypersensitivity, various other contents in different concentrations have been incorporated to dentifrices for treating dentine hypersensitive. Among them, the most frequently used agents are strontium and potassium salts.

\section{Strontium-containing dentifrices}

Strontium-containing dentifrices (especially chloride and acetate) have been studied for almost half a century in the dental literature. ${ }^{35}$ The mechanism associated to strontium utilization is its affinity with dentine and its possible occluding action on dentinal tubules. ${ }^{36}$ The pioneer studies presented promising results, but lacked comparisons. ${ }^{28,37}$ Later, other studies with more adequate methods including comparison groups were performed. Pearce et al. ${ }^{27}$ (1994) in a parallel double-blind clinical trial with 119 patients compared two strontium-containing dentifrices and a fluoride-containing one during 12 
weeks. The authors reported a progressive and significant reduction in dentine hypersensitivity from baseline to the end of the study in the three groups, although without differences among them. West et al. ${ }^{38}$ (1997), also in a parallel double-blind study, compared three dentifrices during 10 weeks. ${ }^{38}$ That study used a strontium acetate dentifrice, a potassium nitrate dentifrice and a control dentifrice only with sodium fluoride. At the end of the study, the three groups presented considerable tooth hypersensitivity effects, without significant differences among the groups. Other studies with similar methodologies $^{3}$ also confirmed these findings and demonstrated that strontium-containing dentifrices are efficacious, although not different from a placebo. The size of the effect attributable to strontium still remains uncertain.

\section{Potassium-containing dentifrices}

The first studies with potassium nitrate for treating dentine hypersensitivity were performed in the 1970's. ${ }^{39}$ Since then, several other studies ${ }^{28,40-42}$ have attempted to analyze the effect of potassium salts (chloride, citrate, oxalate or nitrate) on dentine hypersensitivity. These substances are currently the most studied and sold. The exact mechanism of action of the potassium ions is still uncertain, but it is speculated that high concentrations of potassium close to the dental surface could lead to a depolarization of the membrane of the nerve fibers, preventing their repolarization, thus inhibiting the pain sensation. ${ }^{43}$

Recently, Poulsen et al. ${ }^{44}$ (2006), in a systematic review, presented the results of studies with potassium nitrate based on few individuals (390 volunteers), and concluded that the way hypersensitivity is assessed influences the results. Even if they show promising results, potassium nitrate-based dentifrices still need to be further studied in order to demonstrate their effectiveness.

\section{The placebo effect in dentine hypersensitivity}

As it has been demonstrated in the above-cited studies, a strong placebo effect has been reported in dentine hypersensitivity management. Several fac- tors may contribute to this possible effect. Kienle, Kiene $^{45}$ (1997), point out some aspects that must be considered in a remarkable paper concerning problems in determining the placebo effect.

First, the possibility of spontaneous healing should not be ruled out. Dentine hypersensitivity may decrease as time goes by, since the deposition of reparative dentine could protect the pulp from further stimuli, and since studies are performed with a time elapse, this could be a possibility. However, some decrease in pain sensation might occur immediately after initiation of the therapy. Second, as previously described, a fluctuation of symptoms is clearly present in cases of dentine hypersensitivity. Different environmental conditions might contribute to this fact. Third, other treatment approaches could be present and confound the results. And, finally, in clinical studies, the answers of politeness and experimental subordination could make individuals report less pain.

However, randomized controlled clinical trials, with double-blind placebo-controlled designs tend to diminish these possible interferences. Although this kind of study has been performed for dentine hypersensitivity, a placebo effect is still present.

This information should be taken into consideration by clinicians, not to prescribe placebos, but to understand that their approach should always be a positive one and with good standards of communication with the patient. This approach will probably account for part of the expected clinical effects. ${ }^{46}$

\section{Clinical management of dentine hypersensitivity}

The present review follows the Consensus-Based Recommendations for the Diagnosis and Management of Dentin Hypersensitivity proposed by the Canadian Advisory Board on Dentin Hypersensivity. ${ }^{47}$ This suggested approach to the clinical management of dentine hypersensitivity is based on its extension in the mouth, which can be classified as localized or generalized. The clinical procedures that should be adopted may be different for the two situations. An isolated, in-office approach can be adopted for the localized form, whereas the use of home-care products by the patient can be a more interesting alterna- 
tive for generalized forms. As the aim of this review was to evaluate home-care products for the treatment of dentine hypersensitivity, more emphasis will be given to this issue.

The main procedure related to the clinical management of dentine hypersensitivity consists in establishing a correct differential diagnosis by the dentist, since the main cause of failure is to treat another condition, such as a caries lesion or an inflammatory condition of the pulp, as dentine hypersensitivity. Thus, the description of pain by the patient must be assessed carefully (short/sharp, pain-inciting stimuli, continuance), in addition to performing an evaluation of the diet (excessive dietary acids, e.g. citrus juices and fruits, carbonated drinks, wines) and gastric acid reflux, combined or not to excessive vomiting.

Once the differential diagnosis is made and a case of generalized dentine hypersensitivity is confirmed, the professional can prescribe a desensitizing dentifrice associated with preventive counseling. Patient participation is primordial, since the recommendations must be followed. These recommendations include suppressing risk factors (biofilm, excessive dietary acids, wine, etc), toothbrushing remote from mealtime, advising against traumatic toothbrushing and guaranteeing compliance in the use of desensitizing agents.

After a 3-4 week period, the patient should be evaluated and, if the condition remains, the professional can complement the therapeutic approach with the prescription of high concentration fluorides as an adjunct to the desensitizing dentifrice. However, if, after 2-3 weeks, pain still persists, a cautious review of the differential diagnosis must be performed. Furthermore, the professional should adopt a positive psychological approach and not use

\section{References}

1. Addy M, Urquhart E. Dentine hypersensitivity: its prevalence, aetiology and clinical management. Dent Update. 1992 Dec;19(10):407-8,10-2.

2. Rees JS. The prevalence of dentine hypersensitivity in general dental practice in the UK. J Clin Periodontol. 2000 Nov;27(11):860-5. all measures at the same time. This way, confidence in the patient-professional relationship is less likely to be lost.

When localized forms of dentine hypersensitivity are present, the professional can use in-office approaches (which are not in the scope of the present paper), including adhesives, laser, oxalates, etc. However, in order to prevent recurrence, indication of anti-hypersensitive dentifrices in these cases is also recommended.

\section{Concluding remarks}

The present non-systematic review of the literature concerning the use of home-care products for treating dentine hypersensitivity has demonstrated that this is a common problem in clinical dentistry which deserves special attention on the part of dental professionals.

In addition to in-office treatments, which have also proven effective (albeit not so different from control approaches), the use of home-care products is an interesting possibility.

To date, it seems that the best cost-effective approach includes the use of anti-hypersensitivity dentifrices, especially those with potassium salts, as an important tool for the management of dentine hypersensitivity. ${ }^{24,44}$ This approach is effective both for the localized and generalized forms of dentine hypersensitivity, although it has been particularly indicated for the latter.

Unequivocal evidence for their use, however, is not available, especially considering the existing placebo effect. This fact puts into the hands of the dental professional the responsibility for adopting the best possible psychological approach for the management of this condition, thus helping patients to live with less or no pain.

3. Chabanski MB, Gillam DG, Bulman JS, Newman HN. Clinical evaluation of cervical dentine sensitivity in a population of patients referred to a specialist periodontology department: a pilot study. J Oral Rehabil. 1997 Sep;24(9):666-72.

4. Orchardson R, Collins WJ. Clinical features of hypersensitive teeth. Br Dent J. 1987 Apr 11;162(7):253-6. 
5. Fischer C, Fischer RG, Wennberg A. Prevalence and distribution of cervical dentine hypersensitivity in a population in Rio de Janeiro, Brazil. J Dent. 1992 Oct;20(5):272-6.

6. Orchardson R, Cadden SW. An update on the physiology of the dentine-pulp complex. Dent Update. 2001 May;28(4):2006,8-9.

7. Davis WB, Winter PJ. Dietary erosion of adult dentine and enamel. Protection with a fluoride toothpaste. Br Dent J. 1977 Aug 16;143(4):116-9.

8. Saxton CA, Cowell CR. Clinical investigation of the effects of dentifrices on dentin wear at the cementoenamel junction. J Am Dent Assoc. 1981 Jan;102(1):38-43.

9. Brannstrom M. The hydrodynamic theory of dentinal pain: sensation in preparations, caries, and the dentinal crack syndrome. J Endod. 1986 Oct;12(10):453-7.

10. Brannstrom M, Garberoglio R. Occlusion of dentinal tubules under superficial attrited dentine. Swed Dent J. 1980;4(3):8791.

11. Guyton A. Textbook of Medical Physiology: Oxford: WB Saunders; 1971.

12. Michelich V, Pashley DH, Whitford GM. Dentin permeability: a comparison of functional versus anatomical tubular radii. J Dent Res. 1978 Nov-Dec;57(11-12):1019-24.

13. Pashley DH, Tao L, Boyd L, King GE, Horner JA. Scanning electron microscopy of the substructure of smear layers in human dentine. Arch Oral Biol. 1988;33(4):265-70.

14. Yoshiyama M, Masada J, Uchida A, Ishida H. Scanning electron microscopic characterization of sensitive vs. insensitive human radicular dentin. J Dent Res. 1989 Nov;68(11):1498502.

15. Susin C, Haas AN, Oppermann RV, Haugejorden O, Albandar JM. Gingival recession: epidemiology and risk indicators in a representative urban Brazilian population. J Periodontol. 2004 Oct; $75(10): 1377-86$.

16. von Troil B, Needleman I, Sanz M. A systematic review of the prevalence of root sensitivity following periodontal therapy. J Clin Periodontol. 2002;29 Suppl 3:173-7; discussion 95-6.

17. Prati C, Montebugnoli L, Suppa P, Valdre G, Mongiorgi R. Permeability and morphology of dentin after erosion induced by acidic drinks. J Periodontol. 2003 Apr;74(4):428-36.

18. Suge T, Kawasaki A, Ishikawa K, Matsuo T, Ebisu S. Effects of plaque control on the patency of dentinal tubules: an in vivo study in beagle dogs. J Periodontol. 2006 Mar;77(3):454-9.

19. Kawasaki A, Ishikawa K, Suge T, Shimizu H, Suzuki K, Matsuo $\mathrm{T}$ et al. Effects of plaque control on the patency and occlusion of dentine tubules in situ. J Oral Rehabil. 2001 May;28(5):439-49.

20. Kuroiwa M, Kodaka T, Kuroiwa M, Abe M. Dentin hypersensitivity. Occlusion of dentinal tubules by brushing with and without an abrasive dentifrice. J Periodontol. 1994 Apr;65(4):291-6.
21. Hewlett ER. Etiology and management of whitening-induced tooth hypersensitivity. J Calif Dent Assoc. 2007 Jul;35(7):499506.

22. Narhi M, Kontturi-Narhi V, Hirvonen T, Ngassapa D. Neurophysiological mechanisms of dentin hypersensitivity. Proc Finn Dent Soc. 1992;88 Suppl 1:15-22.

23. Narhi M, Yamamoto H, Ngassapa D, Hirvonen T. The neurophysiological basis and the role of inflammatory reactions in dentine hypersensitivity. Arch Oral Biol. 1994;39 Suppl:23S30 s.

24. Kanapka JA. Over-the-counter dentifrices in the treatment of tooth hypersensitivity. Review of clinical studies. Dent Clin North Am. 1990 Jul;34(3):545-60.

25. West NX. Dentine hypersensitivity: preventive and therapeutic approaches to treatment. Periodontol 2000. 2008;48:31-41.

26. Pamir T, Ozyazici M, Baloglu E, Onal B. The efficacy of three desensitizing agents in treatment of dentine hypersensitivity. J Clin Pharm Ther. 2005 Feb;30(1):73-6.

27. Pearce NX, Addy M, Newcombe RG. Dentine hypersensitivity: a clinical trial to compare 2 strontium densensitizing toothpastes with a conventional fluoride toothpaste. J Periodontol. 1994 Feb;65(2):113-9.

28. Schiff T, Dotson M, Cohen S, De Vizio W, McCool J, Volpe A. Efficacy of a dentifrice containing potassium nitrate, soluble pyrophosphate, PVM/MA copolymer, and sodium fluoride on dentinal hypersensitivity: a twelve-week clinical study. J Clin Dent. 1994;5 Spec No:87-92.

29. Bezerra de Menezes LM, Volpato MC, Rosalen PL, Cury JA. Bone as a biomarker of acute fluoride toxicity. Forensic Sci Int. 2003 Nov 26;137(2-3):209-14.

30. Fejerskov O, Manji F, Baelum V. The nature and mechanisms of dental fluorosis in man. J Dent Res. 1990 Feb;69 Spec No:692-700; discussion 21.

31. Docimo R, Montesani L, Maturo P, Costacurta M, Bartolino $\mathrm{M}, \mathrm{DeVizio} W$ et al. Desensitizing efficacy of a new toothpaste containing $5.5 \%$ potassium citrate: a 4 -week clinical study. Am J Dent. 2007 Aug;20(4):209-11.

32. Merika K, Heftit Arthur F, Preshaw PM. Comparison of two topical treatments for dentine sensitivity. Eur J Prosthodont Restor Dent. 2006 Mar;14(1):38-41.

33. Ritter AV, de L Dias W, Miguez P, Caplan DJ, Swift EJ Jr. Treating cervical dentin hypersensitivity with fluoride varnish: a randomized clinical study. J Am Dent Assoc. 2006 Jul;137(7):1013-20; quiz 29.

34. Yates RJ, Newcombe RG, Addy M. Dentine hypersensitivity: a randomised, double-blind placebo-controlled study of the efficacy of a fluoride-sensitive teeth mouthrinse. J Clin Periodontol. 2004 Oct;31(10):885-9.

35. Ross M. Hypersensitive Teeth: effect of a strontium chloride in a compatible dentifrice. J Periodontol. 1961;32:49-53.

36. Banfield N, Addy M. Dentine hypersensitivity: development and evaluation of a model in situ to study tubule patency. J Clin Periodontol. 2004 May;31(5):325-35. 
37. Skurnik H. Control of dental hypersensitivity: Preliminary report on a strontium containing dentifrice. J Periodontol. 1963;34:183.

38. West NX, Addy M, Jackson RJ, Ridge DB. Dentine hypersensitivity and the placebo response. A comparison of the effect of strontium acetate, potassium nitrate and fluoride toothpastes. J Clin Periodontol. 1997 Apr;24(4):209-15.

39. Hodosh M. A superior desensitizer - potassium nitrate. J Am Dent Assoc. 1974 Apr;88(4):831-2.

40. Conforti N, Battista GW, Petrone DM, Petrone ME, Chaknis $\mathrm{P}$, Zhang YP et al. Comparative investigation of the desensitizing efficacy of a new dentifrice: a 14-day clinical study. Compend Contin Educ Dent Suppl. 2000(27):17-22; quiz 8.

41. Nagata T, Ishida H, Shinohara H, Nishikawa S, Kasahara S, Wakano $\mathrm{Y}$ et al. Clinical evaluation of a potassium nitrate dentifrice for the treatment of dentinal hypersensitivity. J Clin Periodontol. 1994 Mar;21(3):217-21.
42. Silverman G. The sensitivity-reducing effect of brushing with a potassium nitrate-sodium monofluorophosphate dentifrice. Compend Contin Educ Dent. 1985 Feb;6(2):131-3,6.

43. Kim S. Hypersensitive teeth: desensitization of pulpal sensory nerves. J Endod. 1986 Oct;12(10):482-5.

44. Poulsen S, Errboe M, Lescay Mevil Y, Glenny AM. Potassium containing toothpastes for dentine hypersensitivity. Cochrane Database Syst Rev. 2006;3:CD001476.

45. Kienle GS, Kiene H. The powerful placebo effect: fact or fiction? J Clin Epidemiol. 1997 Dec;50(12):1311-8.

46. Lier BB, Rosing CK, Aass AM, Gjermo P. Treatment of dentin hypersensitivity by Nd:YAG laser. J Clin Periodontol. 2002 Jun;29(6):501-6.

47. Consensus-based recommendations for the diagnosis and management of dentin hypersensitivity. J Can Dent Assoc. 2003 Apr;69(4):221-6. 